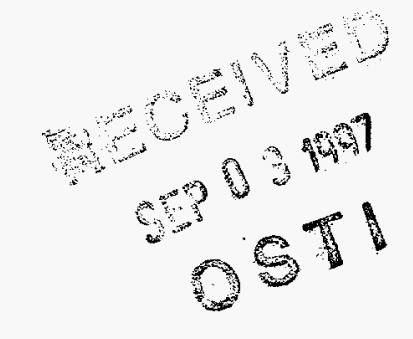

\title{
Intelligent Tools and Process Development for Robotic Edge Finishing: LDRD Project Final Report
}

Christopher L. Lewis

\section{Prepared by}

Sandia National Laboratories

Albuquerque, New Mexico 87185 and Lyermore, California 94550

Sandia is a multiprogram laboratory opeerated by Sahda Corporation, a Lockheed Martin Company, for the United States Departiment of Energy under Contract DE-AC04-94AL 85000.

Approved for public release; distribution is unlimited.

\section{Sandia National Laboratories \\ K}
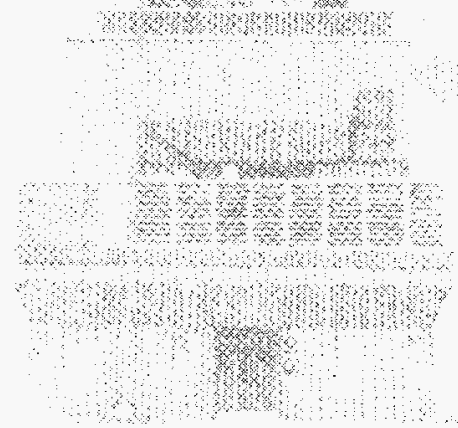

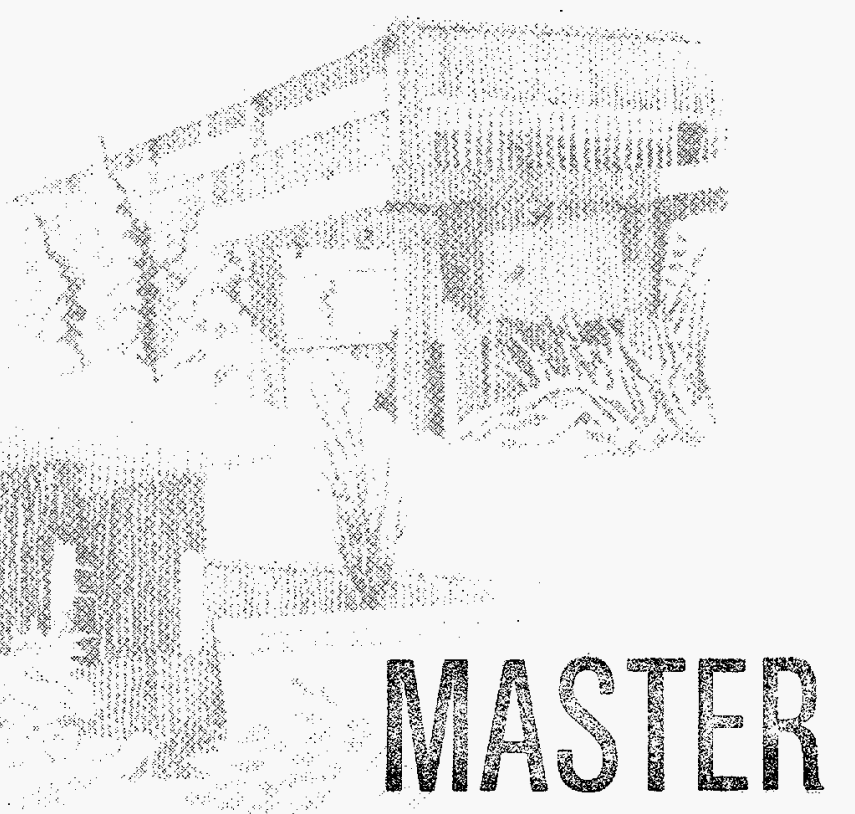


Issued by Sandia National Laboratories, operated for the United States Department of Energy by Sandia Corporation.

NOTICE: This report was prepared as an account of work sponsored by an agency of the United States Government. Neither the United States Government nor any agency thereof, nor any of their employees, nor any of their contractors, subcontractors, or their employees, makes any warranty, express or implied, or assumes any legal liability or responsibility for the accuracy, completeness, or usefulness of any information, apparatus, product, or process disclosed, or represents that its use would not infringe privately owned rights. Reference herein to any specific commercial product, process, or service by trade name, trademark, manufacturer, or otherwise, does not necessarily constitute or imply its endorsement, recommendation, or favoring by the United States Government, any agency thereof, or any of their contractors or subcontractors. The views and opinions expressed herein do not necessarily state or reflect those of the United States Government, any agency thereof, or any of their contractors.

Printed in the United States of America. This report has been reproduced directly from the best available copy.

Available to DOE and DOE contractors from

Office of Scientific and Technical Information

P.O. Box 62

Oak Ridge, TN 37831

Prices available from (615) 576-8401, FTS 626-8401

Available to the public from

National Technical Information Service

U.S. Department of Commerce

5285 Port Royal Rd

Springfield, VA 22161

NTIS price codes

Printed copy: A03

Microfiche copy: A01 


\section{DISCLAMMER}

Portions of this document may be illegible in electronic image products. Images are produced from the best available original docoment. 


\title{
Intelligent Tools and Process Development for Robotic Edge Finishing: LDRD Project Final Report
}

\author{
Christopher L. Lewis \\ Intelligent Systems and Robotics Center \\ Sandia National Laboratories \\ P.O. Box 5800 \\ Albuquerque, NM 87185-1003
}

\begin{abstract}
This report describes a project undertaken by Sandia National Laboratories to develop an agile automated, high-precision edge finishing system. The project involved re-designing and adding additional capabilities to an existing finishing work-cell. The resulting work-cell may serve as a prototype for production systems to be integrated in highly flexible automated production lines. The system removes burrs formed in the machining process and produces precision chamfers. The system uses an expert system to predict the burr size from the machining history. Within the CAD system tool paths are generated for burr removal and chamfer formation. Then, the optimal grinding process is automatically selected from a database of processes. The tool trajectory and the selected process definition is then down-loaded to a robotic control system to execute the operation. The robotic control system implements a hybrid fuzzy logic-classical control scheme to achieve the desired performance goals regardless of tolerance and fixturing errors. This report describes the system architecture and the system's performance.
\end{abstract}


Intentionally Left Blank 


\section{Introduction}

Since the late 1980's, edge finishing processes have seemed like ideal candidates for automation. Most edge finishing processes are unpleasant, dangerous, tedious, expensive, unreliable, and labor intensive. Estimates place the cost of manual edge finishing processes at $12 \%$ of the total cost of fabricating precision parts [1]. For small, high precision parts, the cost of hand-finishing may be as high as $30 \%$ of the total part cost [2]. Up to $50 \%$ of this cost could be saved through automation [3]. This cost estimate includes the direct costs of edge finishing: the machining hours required and the $30 \%$ scrap and rework rate after manual finishing. Ignored are the indirect costs that are not tracked by most production organizations: increased medical costs due to cumulative trauma disorders caused by the repetitiveness of edge finishing motions, the retraining costs caused by high turnover in finishing jobs, and lost opportunity costs by workers who could be employed in value-adding pursuits [4].

Despite the apparent advantages, edge finishing has defied automation in US Industry except in low precision and very high volume production environments. Closer to home, finishing automation systems have not been deployed successfully for DOE Defense Programs (DP) production. A few systems have been attempted [5], but have been subsequently abandoned for the traditional edge finishing method: scraping, grinding, and filing the edges using modified dental tools and hand held power tools.

Edge finishing automation has been an elusive, but still potentially lucrative production enhancement. The barriers preventing the automation of edge finishing processes for medium to low volume, high precision, agile production have proven difficult to overcome. The amount of time required for mechanical reconfiguration of the work-cell for new parts, the time required for reprogramming the work-cell to finish new parts, and the inability of the automation equipment to respond to fixturing errors and part tolerances are the most common reasons cited for eliminating edge finishing as an option for agile production. Existing automated finishing systems have proven to be economically viable only where setup and reprogramming costs are a negligible fraction of overall production costs [4].

Attacking the problems a two-year LDRD effort entitled "Intelligent Tools and Process Development for Robotic Edge Finishing" was initiated in FY95 to address the issues preventing the automation of agile edge finishing processes. The LDRD goal was to develop the technologies needed for agile work-cell reconfiguration and re-programming, and for smart process development. The LDRD was completed in September 1996 and it met all of its technical goals. The technology development encompassed creating a true software connection between the design and fabrication functions so that design engineers can view the consequences of design decisions on fabrication, developing off-line process development and verification capabilities, and developing robust real-time process control technology for edge finishing. 


\section{System Overview}

We developed an automated robotic edge finishing system that is fully integrated into the CAD-CAM system. Within the CAD system the component designer specifies the machining process to be used to fabricate the part. Similarly the edge finishing tool paths are also generated. Then an expert system is invoked to predict the size and shape of the burrs produced by the machining processes. A database characterizing the material removal rate and tolerance capabilities of a variety of edge finishing tools as a function of feed rates and applied forces is then used to select the optimal edge finishing tool and to specify the process parameters of feedrate and force along the finishing trajectory. The manufacturing process information is then exported from the CAD system and the part is fabricated. The finishing operations are performed in a robotic work-cell. The real-time controller that was developed for this purpose automatically adjust the nominal CNC trajectory to fixturing and tolerance variations while maintaining the feed rates and force parameters necessary to remove the burr and to form the desired chamfer.

The robotic edge finishing system enhanced existing system capabilities and greatly increase the number of applications that can be successfully automated. Safety, cost, and quality benefits can be realized by automation, relieving the DOE/NWC and U.S. companies from undesirable, dirty, noisy, and hazardous manual edge finishing processes.

\section{System Architecture}

The rapid programming and process development barriers were attacked through the development of a modular system architecture. The architecture was designed as a set of four stand-alone programming modules that interact through the use of common file formats. The modular approach was used so that the various modules could be reused in other systems and systems could be customized if some of the features were not needed. All four programming modules (low-level control, path verification, process development, and burr prediction) run under the Pro ENGINEER menu structure and operate on Pro ENGINEER defined part files and Pro MANUFACTURE defined manufacturing process files.

The system architecture was designed to allow a Pro ENGINEER generated part definition and a Pro MANUFACTURE definition of the fabrication process (that was used to create the part) to be electronically imported into the work-cell. By working through the four programming modules a viable finishing process can be chosen to produce the desired edge condition; the tool path can be defined and verified; the required process parameters can be determined; and the defined process can be down-loaded and executed by an automated platform. A block diagram of the architecture is shown in Figure 1. The first three modules are fully integrated into Pro/ENGINEER and Pro/MANUFACTURING (the DOE/DP standard CAD/CAM system) using the Pro/DEVELOPMENT environment. The real-time control software resides on the Adept robot's native controller. The modules were designed to operate independently. Information is passed between modules using 
simply formated text files or standard machine code such as cutter location(CL) files.

Two databases of information are required for the system to function. The first database contains the burr formation models for the fabrication processes. This database is used to evaluate the fabrication processes and determine the location and size of burrs that will be present on the as-machined part. The size and location information is used to determine the volume of material that must be removed from the part edge. The removal volume and the required edge tolerance are used in the process planning software in conjunction with the second database. The second database contains empirically-derived finishing process models. The database is queried to pick a viable process and to calculate the process parameters (tool feedrate and tool cutting force) that will produce the desired final edge condition. A hyper-point data structure is used to store, load, and execute the tool trajectory and process definition. A hyper-point is defined as a 14-channel data point that includes the tool location, tool orientation, desired edge dimensions, predicted as-machined edge condition, and the process definition. The programming modules input the hyper-points, add additional information to the hyper-points, and output a hyper-point file that is ready for the next module in the system.

The robotic work-cell was outfitted as shown in Figures 2 and 3. The wrist has a 6DOF force sensor for feedback. The work bench is equipped with a modular fixturing system so that the parts can be positively located and held rigidly. There is a four location tool rest, and the wrist has a quick release tool changer, so that tools are interchanged automatically. These mechanical features allow the work-cell to be reconfigured for new parts and or new tooling very rapidly.

The following sections describe the development and operation of the individual modules. The Burr Prediction and Process Selection modules rely heavily on process characterizations. The methods used in characterizing the milling and chamfering processes will be described first.

\section{Process Characterization}

In conjunction with the University of California, Berkeley, a software module was developed that analyzes face milling processes, as defined by CL Data file output from Pro MANUFACTURE, and predicts the burr sizes and locations on the part. The burr size information is then appended to the tool trajectory path points as extra channels of data defined in a hyper-point data structure. The burr prediction module is currently available for multi-pass face milling operations. Extensions are possible for other types of end mills, side-mills, and ball mills. New mills should be characterized using the process outlined in this section.

Burr height and thickness have been shown to vary in relation to several cutting parameters such as in-plane exit angle, axial depth of cut, and feed as well as tool geometry. An experiment was run to characterize the burr's dependency on these variables. This section describes a general scheme to generate burr data under various parameters during an end milling or face milling process. This plan was created with SS304L workpieces and a 


\section{System Architecture}

\section{Pro/Engineer}
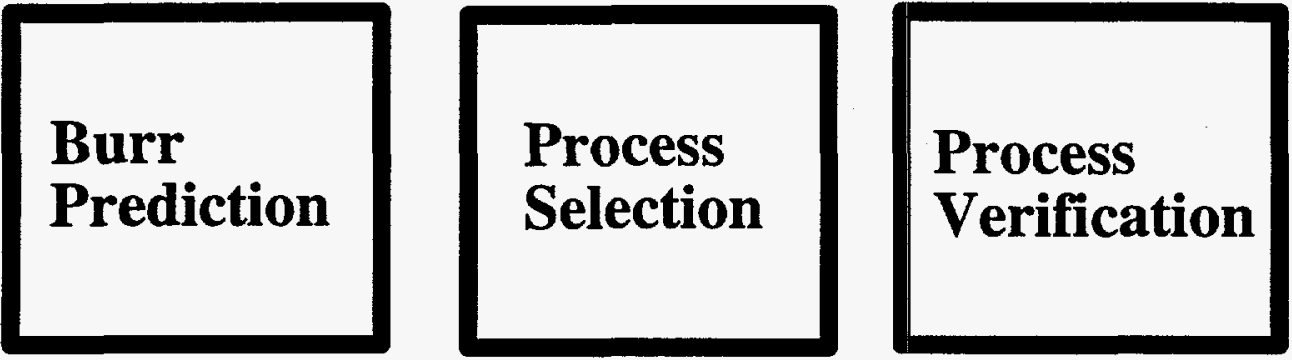

\section{Real Time Control}

Figure 1: The edge finishing system consist's of four-stand alone software modules, three of which are integrated into Pro/Engineer. The burr prediction module is an intelligent system the analyses the Pro/Manufacturing machining process file to predict the burr formation. The process selection module analyzes the edge-finishing tool path and the predicted burr size to select the appropriate burr removal process. The process verification module ensures that the robot will be able to traverse the tool path. The real time control module executes the edge finishing tool path and automatically adjusts for fixturing and tolerance errors. 


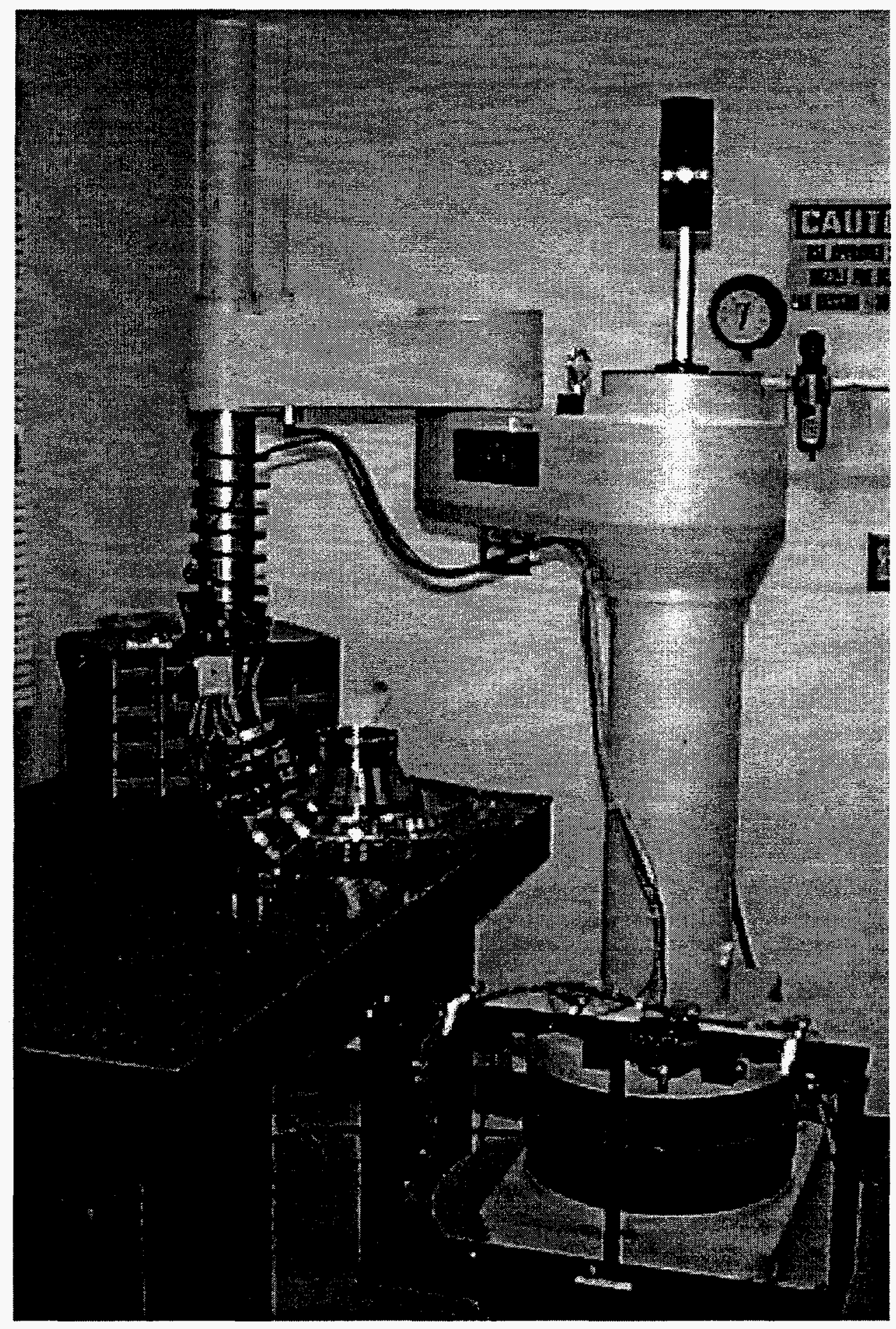

Figure 2: The edge finishing work-cell consists of an Adept robot, a work bench, and a tool rest. CNC tool paths are down-loaded to the controller and executed by the real-time software module. A force sensor provides feedback to adjust for fixturing and tolerance variations. 


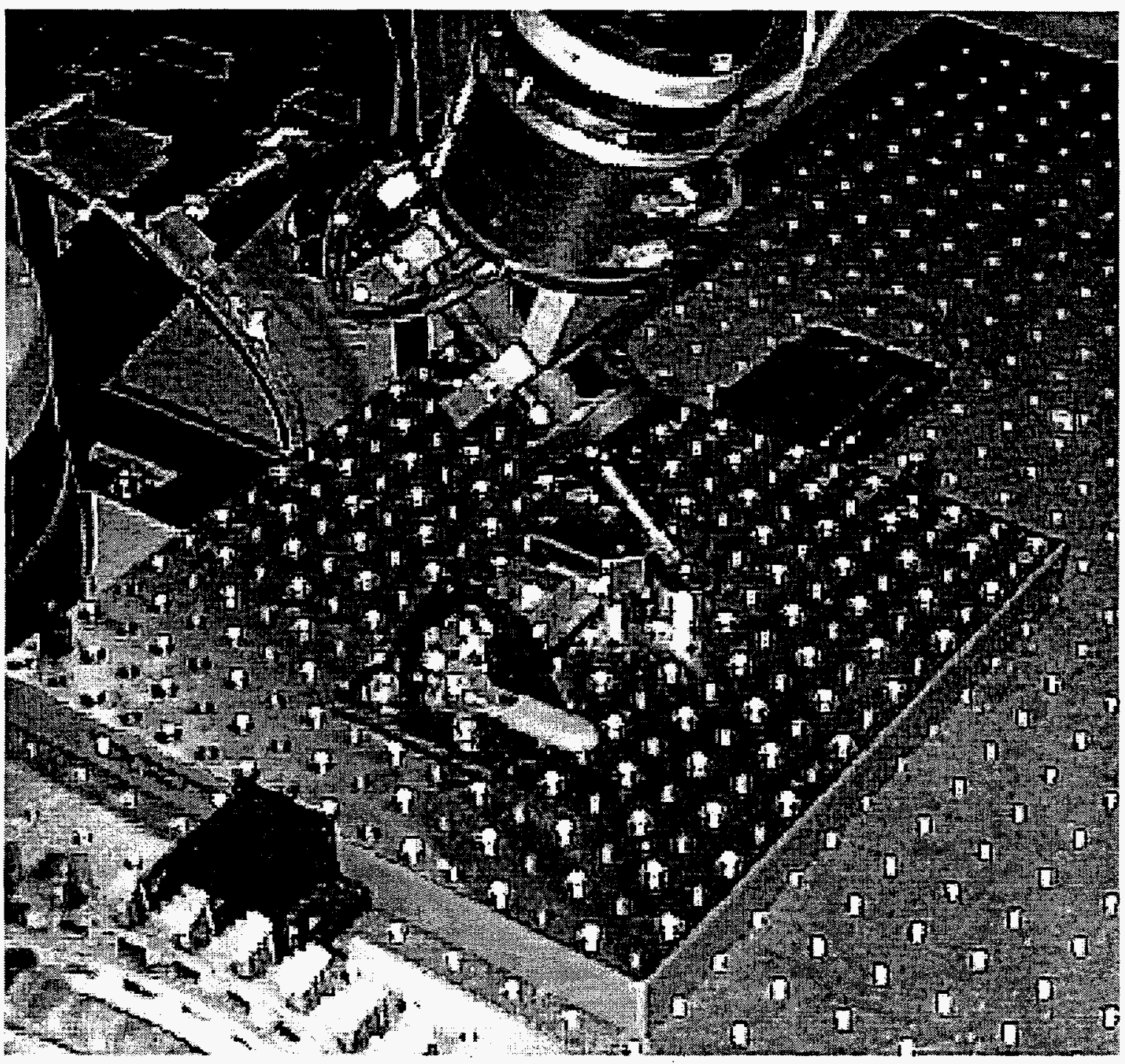

Figure 3: A variety of pneumatic deburring tools and bits remove the burr and form the chamfer. A force sensor mounted on the wrist provides feedback for closed loop control. A modular fixturing system positively locates and secures the part.

specific end mill in mind, however, it can be easily "scaled" for various tool diameters and any suitable material. The formulae used to correlate burr location and in-plane exit angle were derived in generality and can therefore be applied to any experiment derived from this process plan.

The experimental parameters and constant factors are listed in Tables 1 and 2. A total of 108 cuts are required for this series of tests:

2 Cutting Speeds $\times 3$ Feeds $\times 3 D O C^{\prime} s \times 2 \Delta_{\Psi} \times 3$ Repetitions $=108$ cuts. 
Table 1: Test Parameters

\begin{tabular}{|c|c|}
\hline Parameter & Multiple Levels \\
\hline \hline Cutting Speed (CS) & 40,80 sfpm \\
\hline Feed per Tooth (Ft) & $0.004,0.0065,0.009$ inch \\
\hline Axial Depth of Cut (DOC) & $0.030,0.140,0.250$ inch \\
\hline Gradient & Positive, Negative \\
\hline
\end{tabular}

Table 2: Fixed Parameters

\begin{tabular}{|c|c|}
\hline Parameter & Description \\
\hline \hline Tool Geometry & 4-fluted general purpose end mill 1" diameter regular helix \\
\hline Tool Material & High Speed Steel (HSS) \\
\hline Workpiece Material & Stainless Steel 304L (Annealed) \\
\hline
\end{tabular}

With 2 cuts per specimen, a total of 54 workpiece specimens are required. Tables 1 and 2 identify the exact cutting conditions (spindle speed, feedrate, etc) and the fixed parameters.

A total of 3 workpiece specimens were used. While the depth of cut and feedrate are changed for each workpiece, the variation of in-plane exit angle is accommodated by the angle of the tool path with respect to the edge of the workpiece. The workpiece geometry is essentially a rectangular block with dimensions that depend on the diameter of the end mill and the tool path. For this experiment, the workpiece dimensions are: 2.25 " $W \times$ 3.00 " $L \times 1.00 " H(57.2 \times 76.2 \times 25.4 \mathrm{~mm})$. The height of the workpiece can vary depending on the specifications of the vise used. A minimum height of 1.00 " is specified for these tests; however, the height need only be large enough to provide sufficient gripping area for the vise to securely hold the workpiece. Tolerances on the width and length dimensions are not critical, and thus may lie within 0.02 of an inch. Orthogonality of all four sides and the top surface should be kept to within $10^{\circ}$ Assuming that the stock material meets these specifications (otherwise finishing cuts should be performed on the workpiece), the top side of the workpiece must be milled with a finishing cut (DOC approximately 0.030 inch) to ensure a flatness of $0.002 \mathrm{in.}$ The preparation of workpieces typically occurs right before the actual test cut so that the tool's orientation with respect to the workpiece can be retained. Also, it is imperative that the edges where burrs will be created are initially burr-free. Any burrs on these edges, from finishing cuts, should be manually filed down to produce a "clean," non-chamfered edge.

The tool path is specified as follows. The path of the tool remains at a fixed angle $20^{\circ}$, with respect to the workpiece edge. There are also two segments of the tool path that will cause the in-plane exit angle to either increase or decrease as the tool moves through the 
workpiece. This parameter (either increasing or decreasing) has been shown to influence burr size in similar machining processes and is of interest to researchers [3]. The actual tool path is specified by three points, $A, B$, and $C$. On each workpiece, decreasing and increasing (gradients are implemented along the two paths $\mathrm{AB}$ and $\mathrm{BC}$, respectively.

The experimental procedures for the tests are listed below to summarize the process plan.

1. Secure the workpiece in the vise.

2. Mill the top surface of the workpiece with a finishing cult (DOC approximately 0.030 inch). Before repositioning the tool, record its position or "zero" the vertical axis of the NC machine so that a proper depth of cut can be obtained.

3. Inspect and manually remove any burrs present on the critical edges. When deburring, care should be taken to avoid any chamfering (the angle at these edges should be as close to $90^{\circ}$ as possible).

4. Program the A, B, and C points of the tool's center with respect to the origin. From the a top view, the origin is located at the lower left corner of the workpiece.

5. Program the cutting parameters (spindle speed, feedrate, and depth of cut). Refer to the Experimental Array (Appendix D) and enter the proper cutting parameters.

6. Execute the cut.

7. Carefully remove workpiece and rinse the specimen with alcohol to wash off coolant residue taking care not to disturb the burrs.

8. On the workpiece, attach a small self-adhesive label and clearly mark the corresponding specimen number (i.e., 1-54) not the Condition number.

9. Inspect tool, and replace if failure has obviously occurred (i.e., chipped tooth) or after every 6 specimens are completed. Attach another label to the tool, and indicate the specimen numbers it was used to cut. Save all tools.

10. Record comments. In the last column of the Experimental Array, record any tool changes or abnormal occurrences. Repeat from (1).

\section{Burr Prediction Module}

The purpose of the Burr Prediction Module is to predict the burr size and shape conditions along the part edge. The algorithm processes a sequence of hyper-points representing the edge path. When significant changes in burr properties occur relative to the tolerance of the desired chamfer depth, a new hyper-point is inserted into the deburring trajectory. Each hyper-point specifies the tool's path, feedrate and required force. The burr prediction 
module uses the part design CAD file (*.prt), the part machining process plan CL data file (*.ncl), and the Process Definition file (*.ifp) to produce the hyper-point trajectory file. Like all the modules, the Burr Prediction Module is called via menu-selection from Pro/ENGINEER. The module graphically displays the hyper-points from the process definition file both before and after the trajectory is processed, so the user can visually validate the paths that are produced. Once the desired results are produced, an updated Process Definition file (*.jpbt) is output to the Process Planning Module. The resulting Process Definition file is in ASCII format, with 14 columns for each row of hyper-point data. The first 9 channels contain the following data: xyzijk (channels 1-6: location and orientation of the deburring tool); move type (channel 7); desired chamfer depth (channel 8); chamfer tolerance (channel 9). The next 3 channels contain burr information described as follows:

- Burr channel 10: BURR HEIGHT (channel value = floating point, units $=\mathrm{mm}$ )

- Burr channel 11: BURR THICKNESS (channel value = floating point, units = mm)

- Burr channel 12: BURR SHAPE (channel value = integer)

\subsection{Pro/Engineer Interface}

Pro/ENGINEER is a commercial computer-aided design (CAD) package which is the comprehensive software platform for the part design process and generation of the part manufacturing plan. The part is designed within Pro/ENGINEER and the CAD design resides in a file with suffix ".PRT". From the part design, the part manufacturing process plan is generated in a format which can be directly utilized by a machine tool using an associated software package called Pro/MANUFACTURE. The process plan is created through interaction with the user and is placed in a file termed a "CL file". Enhancements to Pro/ENGINEER are created using a development software tool called Pro/DEVELOP which allows integration of user-generated software libraries with Pro/ENGINEER. Pro/DEVELOP is used to create the Pro/ENGINEER menu interface component which allows linkage to the Burr Prediction software.

The menu selection bar for BurrEXPERT is added onto the Pro/ENGINEER PART menu through the use of Pro/DEVELOP. Selecting the BurrEXPERT from this menu triggers a call to the C-function "user_burrEXPERT()" which is the entry point to the CAD interface component.

\subsection{Burr Expert}

The CAD interface software is the C-function "user_burrEXPERT()" . The goal of this function is to locate the Process Definition File and the CL machining file for the current part file and to extract eight parameters $(\mathrm{p} 1-\mathrm{p} 8)$ from these three files. These eight parameters and a ninth parameter which is defined within "user_burrEXPERT()" are used 
by the burr prediction software component. This function interacts with the human interface to the Edge Condition Prediction Module and functions as follows:

User prompt: "Enter Process Definition File:" [enter filename] Response: Parses the Process Definition file to extract the data from the first nine channels of the hyper-point data scans which are the only channels with useful information at this point. The data from these channels is written into the data structure called burrExpert hyperPoint and a record of the hyper-points is kept through the use of pointers and the use of the data structure called burrExpert hyperPointList. The pointer to the first hyper-point list element is stored as the ninth parameter for the Burr Prediction software.

After parsing the Process Definition file, the hyper-points for the force-controlled moves are graphically displayed to the user in a planar view. In the hyper-point file channel 7 describes the type of move. Guarded moves, denoted with a 2 in channel 7 move the tooling close to the workpiece while monitoring the force to guard against unintentional contact. Sequences of force controlled moves, denoted with a 3 in channel 7 follow guarded moves. Once the edge is acquired in a sequence of force controlled moves, the desired force and feedrate pair are maintained by the real-time control system.

User prompt: "Enter CL File:" [enter filename] Response: Parses the coupon CL data file which contains the machining parameters for face-milling the coupon. The machining parameters and information extracted from the CL file are:

1. (p1) face-milling tool radius;

2. (p2) tool rotation direction;

3. (p3) depth of cut;

4. (p4) face-milling starting point;

5. (p5) face-milling ending point.

The user is queried to confirm or to enter a new value for each parameter. The sequence of queries is the following:

User prompt: "Enter tool radius:" [return = confirms value displayed is correct] Response: Confirms parsed value of tool radius or inputs new value.

User prompt: "Enter final depth of cut:" [return = confirms value displayed is correct] Response: Confirms parsed value of final depth of cut or inputs new value.

User prompt: "Enter start point $x:$ " [return = confirms value displayed is correct]

User prompt: "Enter start point y:" [return = confirms value displayed is correct]

User prompt: "Enter start point z:" [return = confirms value displayed is correct] Response: Confirms parsed value of face-milling starting point or inputs new value.

User prompt: "Enter end point x:" [return = confirms value displayed is correct]

User prompt: "Enter end point y:" [return = confirms value displayed is correct]

User prompt: "Enter end point z:" [return = confirms value displayed is correct] Response: Confirms parsed value of face-milling ending point or inputs.new value. 
User menu selection: Rotation: CW / CCW [select direction] Response: Sets tool rotation direction.

The current part geometry, which is stored in a part file with suffix ".PRT" will appear on the screen and the user is instructed to select the part surface for the face-milling operation.

User prompt: "Select a location on part surface:" [click on milled surface] Response: Parses the coupon CAD file "current_part.PRT" from Pro/ENGINEER to extract the coupon "edge features" which are the edges on which burrs are expected to form as a result of face-milling the coupon. The edge features include the actual part perimeter edges, as well as any edges on the face of the coupon that result from intersecting features such as the circular edge at the entry of holes in the face of the coupon. Using the design starting point from the file (generically named for the purpose of this document) "current_part.PRT", the remaining parameters used by the Burr Prediction component extracted are: (p6) the coordinates of the exterior corners of the coupon (planar coordinates), (p7) the location of circular islands on the face of the coupon (radius, center), and (p8) the normal vector to the face of the coupon.

The straight part edge points are written as linked lists into the global data structure burrExpert_point and the circular edge radius and center points are written into the global data structure burrExpert_circle. The pointers to the linked lists of points and circles are written into the global data structures burrExpert_pointList and burrExpert_circleList.

The CAD interface software function "user_burrEXPERT()" calls the Burr Prediction software function burrExpert faceMill with 9 parameters. The function call and the nine parameters, referred to and defined as $p 1$ through $p 9$ above, appears as:

$$
\text { burrExpert_faceMill ( } p 1, p 2, p 3, p 4, p 5, p 6, p 7, p 8, p 9) \text {. }
$$

The Burr Prediction Module is responsible for specifying the burr size, shape, and location along the part edges for the selected part surface and for recognizing when a change in burr characteristics along an edge causes the insertion of a new hyper-point. The Burr Prediction system consists of three elements: the BurrEXPERT burr prediction function, the hyper-point insertion function, and the BurrEXPERT Database. The Burr Prediction System is called from the CAD interface component with the part geometry, the coupon machining parameters and the hyper-point list pointer as arguments. The Burr Prediction System operates on two global data structures created by the CAD interface component. The output from the Burr Prediction system is the expanded hyper-point data structure with complete burr specification data which will be utilized by the process planning software to determine the edge-finishing process requirements.

BurrEXPERT isolates each part edge and the hyper-points associated with each edge from the data structures. A coordinate transformation for the hyper-points is performed so that the coordinate system of the vector normal to the face of the part is used to define the locations for burr prediction along the part edge. The BurrEXPERT travels the part edge in-between hyper-points to predict burrs at edge intervals equal to $0.5^{*} \mathrm{~T}$, where $\mathrm{T}$ is the chamfer tolerance from channel 9 of the hyper-point at the beginning of each interval. At each burr location, the in-plane exit angle is computed using the cutting tool geometry and 
the tool path information. The burr height, thickness and shape are predicted by querying the BurrEXPERT Database at each location with the appropriate machining and exit angle information.

The following describe the possible output values in detail.

1. BURR HEIGHT (floating point, units = mm): Burr height is defined geometrically as the distance from the ideal part edge to the maximum extension of the burr, in the direction normal to the part edge. In the case of edge break-out, burr height is negative. The resolution of the burr height will correspond to the resolution of the chamfer tolerance.

2. BURR THICKNESS (floating point, units $=\mathrm{mm}$ ): Burr thickness is defined geometrically as the distance in the plane of the ideal part edge from the point of tangency of the largest inscribed circle (at the root of the burr) to the ideal part edge. The resolution of the burr thickness will correspond to the resolution of the chamfer tolerance.

Burr thickness is defined mechanistically as the distance in the plane of the ideal part edge from the point of the first "observable" plastic deformation of the burr root curvature to the ideal part edge. The deformation shall be observable at $10 \mathrm{X}$.

3. BURR SHAPE (integer): Burr shape defines the cross-sectional appearance of the burr. The currently defined burr shape categories, for which the generic definitions of burr height and burr thickness may be applied, are the following:

4. Knife shape (value $=1$ ): Burr with a right triangular or rectangular cross-section, for which the shape is uniform in the span of two hyper-points, with height remaining constant within a band of $+/-50 \mathrm{~m}$.

5. Burrless (value $=0$ ): No burr present on the edge, because this edge is a region where the cutting tool enters the workpiece.

6. Breakout (value = -1): Edge with negative burr height, in which the ideal corner of the edge has fractured and is no longer attached to the part. The thickness may zero or positive if deformation occurred before the edge corner detached from the part.

Sketches of burr measurement variables and the various burr shapes will be shown in accompanying figures.

7. Hyper-point insertion function A significant change in burr characteristics at a given point along each part edge occurs when the burr height increases or decreases by an amount equal to the one half of the chamfer tolerance $\left(+/-0.5^{*} \mathrm{~T}\right)$ as defined by channel 9 of the current hyper-point channel. When a significance change in the burr height between two hyper-points has occurred, a new hyper-point is created with the new burr properties. The burr height at this hyper-point becomes the basis of comparison for burr changes which trigger the insertion of the next hyper-point. 
The hyper-points will contain three channels defining the predicted burr characteristics:

- Burr channel 10: BURR HEIGHT (channel value = floating point);

- Burr channel 11: BURR THICKNESS (channel value = floating point);

- Burr channel 12: BURR SHAPE (channel value = integer).

\subsection{BurrEXPERT Database.}

The BurrEXPERT Database is constructed from empirical relationships resulting from experimental data collected by face-milling 304L stainless steel. The experimental factors are in-plane exit angle, in-plane exit angle gradient, depth of cut, feed per tooth and cutting speed. A full factorial experimental design is used. The response variables are burr height, burr thickness and burr shape. During database development, the components of the Edge Condition Prediction Module are tested with a data base containing information on burr height only. The burr thickness is predicted assuming that the thickness is equal to one third of the burr height. This relationship was observed experimentally in 304L stainless steel, and provides an estimate of the burr thickness.

The CAD output software reads the hyper-point data from the global hyper-point data structures burr-Expert hyperPointList and burr-Expert.hyperPoint. A new version of the Process Definition file (*.ipbt) is generated in ASCII with updated with hyper-point information. The number of hyper-points has increased according to predicted burr changes, and channels 10 - 12 now contain burr data.

The hyper-points with burr information in the updated Process Definition file are displayed graphically to the user in a planar view. The burr cross-sectional area is computed at each hyper-point using the burr height and burr thickness by assuming that the burr cross-section is right triangular in shape. The amount of burr at each point is displayed using colors which correspond to a color code for the burr cross-sectional area. A small dot represents each hyper-point. If the spatial frequency of the hyper-points exceeds the frequency response capabilities of the force-controlled deburring system, then the tolerance on the change in burr size which triggers a hyper-point insertion can be increased within the BurrEXPERT software or the chamfer tolerance specified may have to be increased.

\section{Process Selection}

Once the burr expert has added the necessary burr size and shape data, the process selection software evaluates the available deburring tooling process characteristics to determine which tools are capable of forming the desired chamfer. A number of dremmel style grinding tools were characterized for the purpose, and the following information was determined.

1. Minimum area, or smallest cross section that can be milled.

2. Maximum area, or largest cross section that can be milled. 
3. Minimum feedrate, at which the tool can operate.

4. Maximum feedrate, at which the tool can operate.

5. Minimum force, that the tool can apply.

6. Maximum force, that the tool can apply.

7. Material Removal rate Polynomial function parameters.

8. Standard Deviation Polynomial function parameters.

The process selection module compares the requirements of each edge to the abilities of each process to determine which tool is capable of producing the desired chamfer. If more than one tool is capable, the algorithm allows the user to select using menu functions.

Once the tool has been selected, the process selection algorithm traverses the edgefinishing tool path and adds the required force and feedrate pair to the hyper-point list. The list is then written to a file.

\section{Process Verification}

Advanced path planning techniques have been implemented under Pro ENGINEER that allow the user to visualize the process, identify collisions, and modify the trajectory to avoid problems that are identified. The process is verified in this virtual environment and prepared for down-loading to the real machine for execution.

The robot work-cell, the flexible fixturing system, and the part are a Pro/Engineer assembly. Menu items are incorporated into the Pro/Engineer environment that allow the user to preview motions of the robot and cutter while it traverses the edge-finishing trajectory. In addition, a traditional robotic teach pendant has been implemented to allow the user to customize trajectories.

\section{Real Time Control}

A new control theory (a low-level hybrid position/force controller with a fuzzy logic-based supervisory controller) has been designed, implemented, and tested on an XY table and ported to an Adept robot. The low-level controller uses position control in the direction normal to the part edge and feedrate-force control in the direction tangent to the part edge. The fuzzy logic supervisor monitors the commanded feedrate and tool cutting forces, and corrects the model-part registration to address sensed anomalies in the process.

A Technical Advance has been filed on the control system and a patent application is pending. Figure 2 shows the control system block diagram. The control system has been tested thoroughly on the XY table and has yielded the following advances in edge finishing control over the classical method of normal force/tangential position control: a) 9611 has 
successfully finished features with smaller radii of curvature than previously possible using an XYZ table (reduction from 0.5 inch to 0.1 inch radius). b) The kinematic instability that occurs when finishing small radius of curvature features using classical hybrid position/force control was eliminated. c) The system precision was increased. The tolerance on chamfered edges was reduced from +0.010 inch to +0.003 inch along a 10 inch path. d) The ability to add additional heuristics to the control system was added to the system through the fuzzy logic supervisor architecture.

\section{Overall System Perfomance}

The modules described above were integrated into Pro ENGINEER and Pro MANUFACTURE and the edge finishing work-cell. To use the system, part models, fixturing design, and part/fixture assembly are all performed in the Pro ENGINEER solid modeling environment. The tool trajectory is then defined using the CNC program generation capabilities of Pro MANUFACTURE. The output from Pro/MANUFACTURE is a CL Data file which is post-processed to generate the initial tool path hyper-points.

To illustrate the versatility of the process, a new part was brought into the work-cell electronically, the fixture reconfigured electronically, the tool path was defined and verified using tool-only animation, the work-cell was mechanically reconfigured, tools were loaded, and the part profile was finished within four hours. Additional time can be shaved off of the re-programming time as our familiarity with Pro MANUFACTURE increases and the program is refined.

\section{Status at the End of the LDRD}

The scope of the LDRD called for a proof-of-concept integrated system to be developed that demonstrates all of the features required of a next generation agile edge finishing system. The pieces of technology that have been discussed currently exist as stand-alone modules. The modules have been integrated under Pro ENGINEER and demonstrated as an integrated system. The software in an Adept work-cell has been modified to incorporate all of the software modules. The low-level control system was integrated into the work-cell. Three finishing processes have been included in the finishing process database and the face milling process has be characterized and is available in the machining database. At this point, the requirements of the LDRD have been satisfied. However, before the system can go into production, the performance of the integrated work-cell will need to be characterized. Benchmarks for reprogramming speed, accuracy and repeatability of the edge dimensions, and the size of features that the system can reliably finish need to be detemined. 


\section{The Next Step: Deploy the Technology}

The advances in automated finishing technology that were developed and demonstrated by the LDRD project "Intelligent Tool and Process Development for Robotic Edge Finishing" attacked all of the existing obstacles to the successful deployment of automated systems. Significant contributions were made in the areas of ease-of-use, system agility, system performance, and knowledge-based process planning. The next logical stage of development takes the system out of the laboratory and places it into the pilot projects. Pilot system deployment would enable performance testing, ease-of-use, and agility to be tested and improved in a production-like environment. The promised benefits from the automation of edge finishing processes were discussed earlier: a 50\% reduction in edge finishing costs which constitute $12-30 \%$ of the total part fabrication cost, a drastic reduction in the $30 \%$ scrap and rework rate after manual finishing, and elimination of the injuries and medical claims resulting from manual finishing.

However, to realize these benefits the technology must be taken out of the laboratory and put into the hands of the people who are responsible for fabricating parts. Significant technical problems exist with the system that need to be identified and must be solved. These problems cannot be identified and addressed in a laboratory setting. The ISRC lacks the experience with manufacturing and product qualification to foresee the problems that production workers will encounter when using an automated finishing system. The project team needs feedback from the field to take the system development effort to the next higher level.

\section{Conclusions}

The automated edge finishing demonstration system has been around for a long time at Sandia. The potential customers for the system recognized the need for additional system development and production hardening of the system and waited for further development to proceed from the early success. However, after the initial demonstration system was completed very few resources were committed to continue the progressive development of the system. The edge finishing LDRD, undertaken in FY95-96, has been a coordinated, well-funded effort designed to move edge finishing beyond the demonstration level of development. The LDRD will result in a proof-of-concept prototype that possesses all of the features that automated, agile edge finishing systems must possess to begin displacing manual finishing as the DP mainstay.

The technologies developed and demonstrated under the edge finishing LDRD will have wider applications outside of automated edge finishing and should not be abandoned. An innovative low-level control system for stable in-contact operations, automated edge finishing process planning, burr prediction from machining scripts, and Pro ENGINEER interfaces are all technologies that can be applied to the problems facing other automation projects within the DP sector. 
The next logical step in the development of automated edge finishing systems is to move the proof-of-concept system from the laboratory to a pilot production environment. Additional problems, features, and enhancements will be identified and addressed in this stage of the development. Sandia must continue to support the technical development of edge finishing systems to begin reaping the benefits promised by the automation of these processes: 6-15reduction in the current 30cumulative trauma disorders attributed to manual finishing. Dropping the technology now will waste the investment Sandia has made in finishing technology development and will ensure that, as long as metal parts are machined for DP applications, people will continue scrapping, filing, and grinding edges using 19th century technology.

\section{References}

[1] SME, Deburring Technology for Improved Manufacturing, Dearborn, MI, 1981.

[2] L. K. Gillespie, "Deburring precision miniature parts," Precision Engineering, pp. 109$116,1979$.

[3] B. R. K. Stouffer, J. Michaloski and F. Proctor, "Adacs - an automated system for part finishing," IECON, vol. v1, 1993.

[4] M. Bruff, "Automated finishing systems market report," .

[5] R. J. Lambrects, "A robotic system for deburring machined castings," June 1986. 


\section{Distribution:}

MS 0188 LDRD Office, 4523

MS 9018 Central Technical Files, 8940-2

(1)

MS 0899 Technical Library, 4916

MS 0619 Review \& Approval Desk, 12690, for DOE/OSTI

MS 1003 Chris Lewis, 9611 